\title{
A VALIDATED INTERVIEW SCHEDULE FOR USE IN POPULATION SURVEYS OF CHRONIC DISEASE AND DISABILITY
}

\author{
JESSIE GARRAD* AND A. E. BENNETT $\uparrow$ \\ Department of Clinical Epidemiology and Social Medicine, St. Thomas' Hospital Medical School, London S.E.1
}

The popular concept of a disabled person is of someone who has lost a limb or who is physically deformed in some obvious way. This concept is largely based on the appreciation of structural damage; severity is considered to be dependent on the extent of the damage and criteria based on this approach govern the assessment and award of the disability pensions at present available. However, disability may be thought of in terms of behaviour and performance and may be considered as limitation of the performance of an individual when compared with a 'fit' person. This concept considers disability as a disorder of function rather than a structural abnormality or loss. In America, reports of the Committee on the Medical Rating of Physical Impairment (1958) distinguish between these two approaches. The concept of the total functional loss is considered to be disability whereas the anatomical or structural abnormality is described as impairment.

We have adopted this distinction but define these two terms more strictly. We define disability as limitation of performance in one or more activities which are generally accepted as essential basic components of daily living, such that inability to perform them necessitates dependence on another person. The severity of disability is thus proportional to the degree of dependence. The areas of activity essential are (1) mobility: walking, negotiating stairs, transfer in and out of bed or chair, and travel; (2) self-care: feeding, dressing, and toilet care; (3) domestic duties: shopping, preparation and cooking of food, household cleaning, and washing of clothes; and/or (4) occupation: the ability to hold unmodified employment in open industry consistent with the individual's age, sex, and skill.

We define impairment as an anatomical, pathological or psychological disorder which may be described in diagnostic or symptomatic terms. It may

\footnotetext{
*Present address: Department of Social Work and Administration, University of Bristol, 6 Berkeley Square, Bristol.

tPresent address: Department of the Regius Professor of Medicine, University of Oxford, 9 Keble Road, Oxford.
}

cause or be associated with disability so that while every disabled person has an impairment, not all people with impairments are necessarily disabled. Impairments may be classified into four categories: those affecting locomotion or any motor activity; those of sensory origin; those referable to internal medicine, e.g., cardiac and respiratory disorders; and those of primarily psychological origin together with unclassifiable organic disorder.

\section{Measurement of Disability}

There are three main methods by which disability defined in functional terms can be identified and measured. The first of these is by clinical assessment of the individual's performance. Unfortunately, however, it has been shown that observers of different professional disciplines use different criteria for assessing performance (Kelman and Willner, 1962). Moreover, perception of change in an individual's performance is related to the observer's role in the treatment and assessment situation (Tamerin, 1964). If an attempt is made to overcome these difficulties by forming a team of several clinical disciplines to make the assessment, unanimity is unlikely. The options then are either to accept democratically the majority opinion, to 'weight' the opinions of the various observers or to accept that the leader of the team has the prerogative to resolve any difference (Kelman and Willner, 1962). These difficulties are more easily resolved in the clinical situation, and the method presents considerable organizational problems for large-scale surveys.

The second principal method involves the use of standard tests of performance by the individual conducted by an observer trained in the administration of the test. Examples are the Maryland Disability Index, also known as the Barthel Index (Wylie and White, 1964) and the Index of Independence in Activities of Daily Living (Index of A.D.L.: Katz et al., 1963). In these scales, a number of activities is defined which an individual must be able to complete unaided in order to live an inde- 
pendent life. The individual's performance is observed and his level of independence in each activity is rated according to carefully defined criteria. In a variation of this method, the individual is tested for his ability to make the basic movements needed to perform these activities rather than his ability to perform the activities (Jefferys, Millard, Hyman, and Warren, 1969).

However, with any tests of performance the problem of the effect of interaction between the individual, the observer, and the test situation is important (Wolf, 1950; Osgood and Suci, 1952; Shontz and Fink, 1957; Cole and Griffith, 1958). Kelman and Willner (1962) obtained three different scores when patients resident in a nursing home were rated under three different sets of conditions: in non-test, day-to-day conditions in the nursing home rated by staff familiar to them; in a test situation in their nursing home rated by staff familiar to them; and in a test situation outside their nursing home rated by unknown staff. In general, the test situation outside the nursing home with unfamiliar observers produced the lowest ratings. However, some patients appeared to be so stimulated by the novelty of the strange situation as to perform better: this phenomenon was also reported by Muller (1961).

Closely associated with the variation in performance due to rapport between individual and observer and the effect of the test situation is the factor of the individual's self-concept. Litman $(1962,1964)$ has shown that patients' progress in rehabilitation is largely dependent on their self-concept. If they consider themselves to be capable of playing a definite, although altered, role in society, they respond better to treatment than if they consider themselves as disabled and limited people. It is arguable that a person's performance in a test situation would be affected in a similar way. Another component of the individual's motivation which may affect performance is the secondary gain which he thinks may result from performing well or badly.

The third method is by questioning the individual as to his current level of daily performance. Communication difficulties and confusional states may render this method impossible or unreliable. For this reason Sett (1963), in his questionnaire, included a section to assess communication and mental state which is administered first and, if necessary, the remainder of the questionnaire is then administered to a relative or member of the nursing staff. When the respondent answers for himself, problems of self-concept and secondary gain are also relevant in this method. While the importance of the latter problem is considerable in the clinical situation it is minimal in research, and the effect of the former problem can be identified by built-in cross-checkso in the interview.

When using an interview technique in research $\frac{3}{\infty}$ surveys the problems of interviewer variation and? interviewer drift arise. The former can be reduced $\overrightarrow{\vec{F}}$ by strict training and supervision and the latter by? periodic check interviews. Of great importance is the effect of the interaction between the interviewer and $\overline{\bar{s}}$. the respondent. Cannell and Marquis (1967), and $\vec{\Phi}$ Cannell, Marquis, and Laurent (1969) have shown that the quantity and quality of data obtained in ${ }^{\infty}$ health surveys is dependent on the attitude and $\overrightarrow{0}$ manner of the interviewer and the style of inter- $-\overrightarrow{-}$ viewing. Again, standardization can be obtained by training and supervision but the possible effect of the interviewer's sex on the validity of the data ? collected should not be overlooked (Colombotos, in Elinson, and Loewenstein, 1968).

\section{DeVelopment of INTERVIEW Schedule}

The prevalence of disability has not been extensively studied and an almost complete lack of anyz knowledge about the size and nature of the problem presents difficulties in planning health and welfare $\vec{\varphi}$ services. For this reason and because of the increasing importance of chronic non-communica disease as a cause of morbidity in middle and adio age we wished to measure the prevalence of dis bility in the population of the catchment area of St. Thomas' Hospital. Comparison of the three methods $\frac{}{\mathrm{D}}$ suggested that the interview was the method of $\stackrel{\circ}{\Rightarrow}$ choice for a large-scale survey conducted in the $\overrightarrow{\overrightarrow{0}}$ respondents' homes. We now describe how a structured interview schedule was developed and tested for this study. Section I of the interview: schedule identifies and assesses the severity of disability and section II classifies the impairment and? identifies the principal diagnostic group. Subsequent $\frac{}{3}$ sections of the schedule, which are not described here, deal with occupational history, family, accom-응 modation, and the use of health and welfare services (schedule available on request).

\section{Section I: Assessment of Disability}

This section was developed in three stages. The $\mathcal{N}_{\circ}$ content was determined by the definition of the essential activities of daily living. Then a series of questions was formulated to identify the respon- $\omega$ dent's maximum level of unaided performance in each activity or, if some help was essential, his maximum performance in each activity with the minimum of help. Criteria to identify disability in ${ }^{?}$ each area of activity and the different levels of $\frac{0}{0}$ severity were at first arbitrarily set from clinical $\frac{\vec{D}}{\mathbb{D}}$ experience. The questionnaire was then tested on $a \frac{\text { P }}{\mathbb{Q}}$ 


\section{SECTION I}

\section{MOBIITY}

Walling Do you walk outdoors in the street (with crutch or stick if used)?
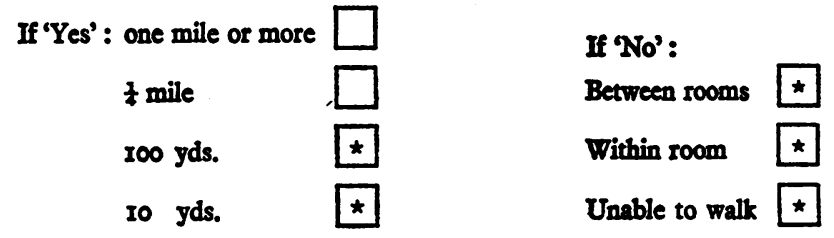

and :

Unaccompanied $\square$

Accompanied $\star \star$

Acc. + support $\star \star$

Stairs Do you walk up stairs ?

To Ist floor or above

5-8 steps or stairs

2-4 steps or stairs

I step

mount stairs other

than by walking

unable to mount stairs $\star$
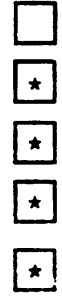

Unacc.

Acc.

Acc. \& Supp.

No need to mount stairs
Do you walk down stairs?

From I floor to another $\square$

5-8 steps or stairs

2-4 steps or stairs

$\star$

$\star$

I step

goes down stairs other

than by walking

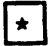

unable to descend stairs $\square$
Unacc.

Acc.

Acc. \& Supp.

No need to descend stairs

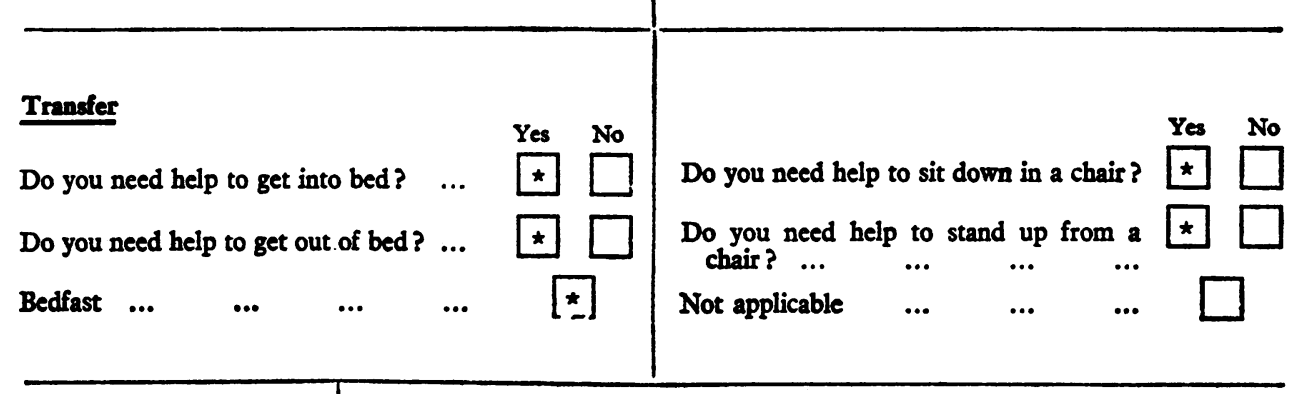

\section{Travel}

Do you drive yourself in a car?

Normal (unadapt.)

Adapted

Invacar

Self-propelled vehicle (outdoors)

Does not drive
Do you travel by bus or train? If 'Yes' : Whenever necessary

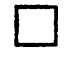

Only out of rush hour

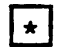
and:

Unaccompanied

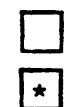

Accompanied
If 'No':

Unable to use bus and train $\star$

Unable to use bus, train and car

$\star$

Does not travel by choice

Uses private transport by choice

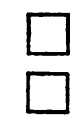


SELP CARE

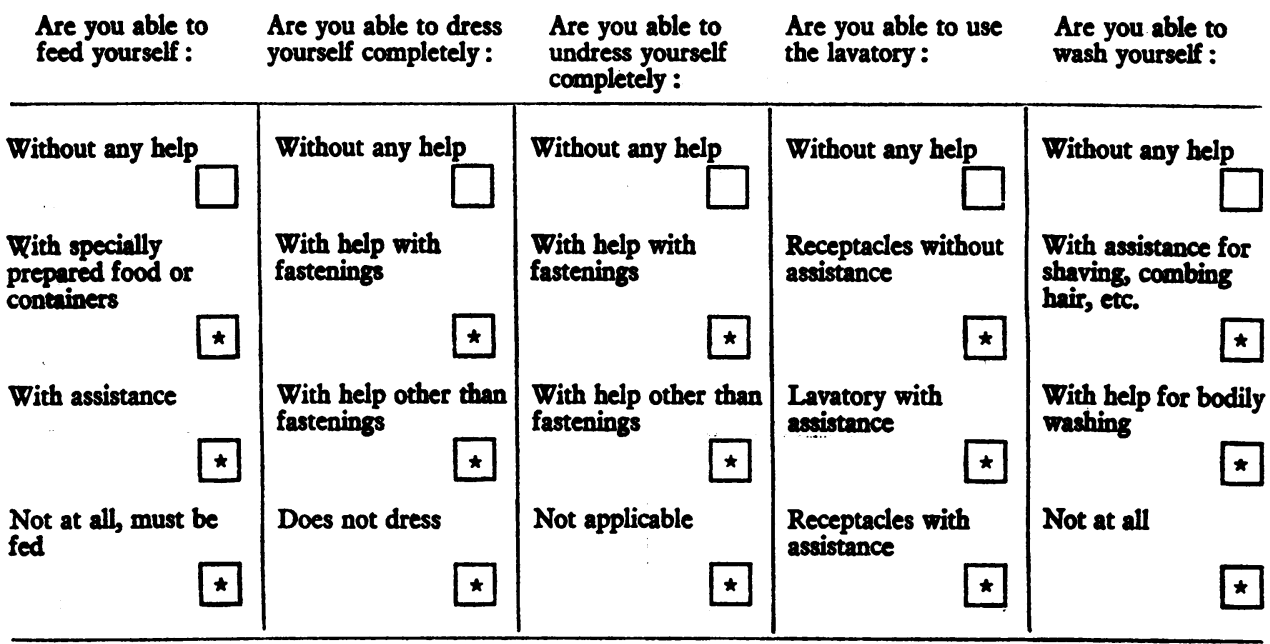

\section{DOMESTIC DUTIES Do you do your own:}

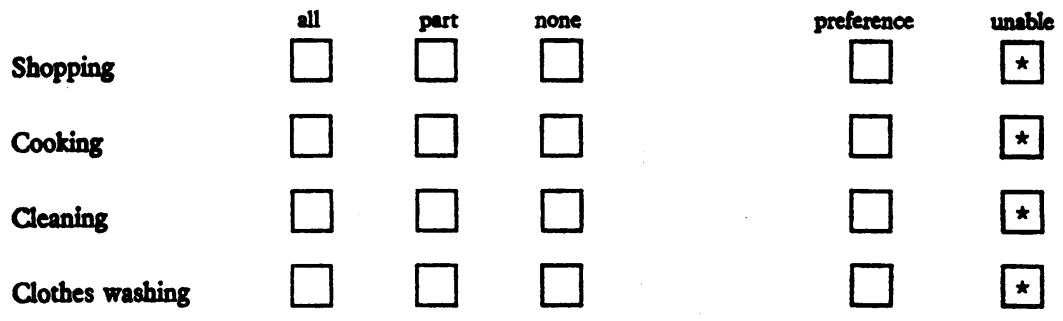

Men with no houschold duties

OCCUPATION Do you have a paid job at present?

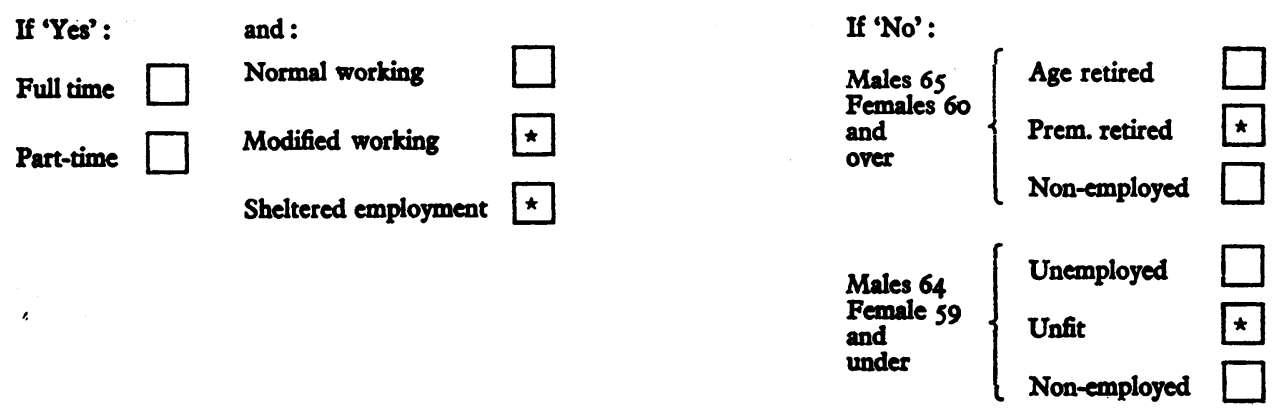


wide range of inpatients and outpatients for meaningfulness, intelligibility, and acceptability. It was repeatedly modified and retested until satisfactory wording and grading were obtained. Finally the layout was designed in a condensed format so that the various subsections were conveniently and clearly set out for the interviewer to complete with the minimum of clerical error (section I of schedule).

Each subsection is introduced by a lead question asking in most instances 'Do you ....?' rather than 'Can you ...?' or 'Could you if ...?' Interviewers were trained to probe to identify the appropriate level of performance. When a respondent's answer fell between two defined levels of performance the higher level was recorded so that disability was underestimated rather than overestimated.

It can be seen that the separate criteria for disability are: walking less than $\frac{1}{4}$ mile unaccompanied; walking up or down less than nine stairs unaccompanied; needing help to get in or out of bed or chair; using an invacar or self-propelled vehicle outdoors; inability to travel by public transport unaccompanied whenever necessary; needing special facilities for feeding; needing assistance with personal washing; inability to perform all the defined domestic duties; inability to undertake unmodified employment if of working age, or having retired prematurely on medical grounds if currently over retirement age. Initially, if an individual met any one of these criteria he or she was classified as disabled. However, it became apparent that all the same criteria could not meaningfully be applied to people aged 65 or over, especially in the area of travel and domestic duties. Therefore, for this age group only, these criteria were modified to accept a lower level of performance as adequate and not indicative of disability. For the majority of men over 65 occupation does not demand the ability to travel on public transport unaccompanied at any time, so this criterion, applicable to the younger age groups, was relaxed. For women over 65 domestic duties become more onerous and many women are not able to maintain their levels of activity. Since there is in the performance of domestic duties a large qualitative component, the criteria for those over 65 were relaxed so that accomplishment of only part of the household duties did not imply disability as it did in the younger age groups.

Scoring presented problems. Many rating scales present a score calculated as an algebraic sum of the component scores. This, however, masks different levels of performance in different areas, results in loss of information, and can be misleading. Also, for the purpose of assessing medical care needs, it is necessary to know in which areas of activity the respondent's performance is deficient so that the appropriate health or welfare service may be selected to assist him.

Ekwall (1966) reviews several methods of assessing disability in which 'socio-medical' and 'psychosocial' factors are considered to be relevant variables. A particular value of his paper lies in a description of the method whereby a large amount of data is quantified and summarized without loss of relevant information. We adopted a similar method of scoring to quantify the level of performance in each essential activity of daily living and designed a method of coding the information for data processing. Each individual is described by four scores, one for each area of essential activity, and these scores may be presented as a profile.

\section{SECTION II: IDENTIFICATION OF IMPAIRMENT}

A locomotor impairment was identified by four questions enquiring for permanent paralysis or weakness of specified parts of the body-by pain and by limitation of movement in specified joints. The interviewer noted tremor of hands and/or speech interference and recorded any comments volunteered by the respondent. A sensory impairment was identified by questions devised to assess loss of hearing, defective vision, loss of tactile sense or appreciation of temperature and disturbance of balance. The subsection for internal impairments consisted partly of the questionnaire on respiratory symptoms (Medical Research Council, 1966) and part of the questionnaire for the diagnosis of ischaemic heart pain (Rose, 1962) with additional questions designed to identify, for example, the presence of diabetes.

A structured subsection enquiring for past medical history and medical care usage in the previous nine months often provided confirmation of the impairment identified by the preceding sections. Respondents suffering an impairment which could not be confidently allocated to one of the first three categories were allocated to the fourth category of 'other' together with those whose impairment was clearly of psychological origin. A psychological screening questionnaire (Foulds, 1965) was included in section II to give a measure of 'personal disturbance'.

These two different sections were reworded and retested until they were intelligible to a sample of patients, and the data obtained broadly corresponded with information available in the medical notes. The design was amended until the lay-out was convenient to the interviewer.

\section{VALIDATION}

The validity of these two sections was measured 
by comparison with clinical assessments. The Departments of Geriatrics and of Physical Medicine maintain detailed records of the patients' performance and much additional data and social information are available in the records of the medical social workers. Fifty-two outpatients attending these departments were selected for us by the staffs of the departments. These patients were seen at home by trained interviewers who had no prior knowledge of the patients. Before the interview was started it was carefully explained that it was part of a research programme and not part of treatment. The data recorded in the completed interview schedules were then compared with the medical and social work records.

The patients, 17 men and 35 women, were aged between 35 and 74 years. The clinical records show that five were impaired only, five disabled in one area of essential activity, 18 in two areas, 19 in three areas, and five in all four areas. The primary diagnoses of the 52 patients were: rheumatoid arthritis, arthritis, etc., 22; cerebrovascular disease, 9; neurological disease including sensory disorders, 10; cardiovascular, respiratory, and other internal disorders, 10. In one patient the primary diagnosis was psychological disorder but the patient also suffered from cervical spondylosis.

Table I shows the agreement of the completed interview schedule with the clinical record. In all cases the disability status was correctly described. Two errors occurred in allocation to the impairment category and three errors in identification of the primary diagnosis. These five errors concerned three patients. The impairment category of the patient with psychological disorder was incorrectly assigned as a result of the diagnosis of spondylosis; no diagnosis of psychological disorder was made. A diagnosis of congestive cardiac failure and allocation to the category of internal impairment were incorrect in a patient suffering from arthritis, obesity, and diabetes. In the third patient the correct diagnosis of osteoporosis was not made and cerebrovascular disease was diagnosed. The clinical records of the 52 patients revealed disability in a total of 118 areas

TABLE I

VALIDATION OF INTERVIEW SCHEDULE

(Agreement of the interview schedule with the clinical record in assigning the respondent to the correct disability status, impairment category, and diagnosis)

\begin{tabular}{l|c}
\hline & $\begin{array}{c}\% \text { Correctly Assigned } \\
(\mathbf{n}=52)\end{array}$ \\
\hline $\begin{array}{l}\text { Disability status } \\
\text { Primairment category }\end{array}$ & 100 \\
\hline
\end{tabular}

TABLE II

RELIABILITY OF INTERVIEW SCHEDULE

(Comparison of assessments made one year apart by different interviewers on 153 respondents)

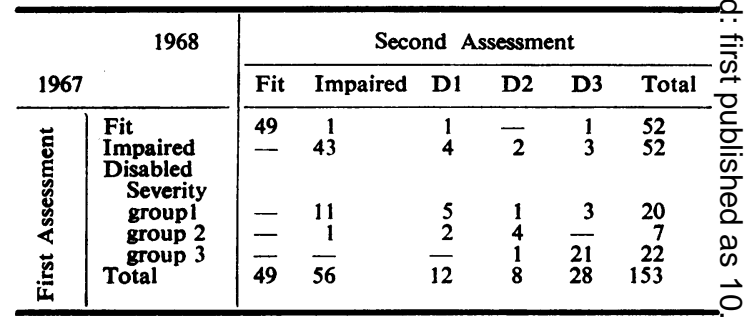

and of these $108(91.5 \%)$ were recorded at interview $\stackrel{\omega}{\circ}$ In addition to these 10 false negatives, the interview schedules contained one false positive identification of disability in the area of occupation.

\section{RELIABILITY}

The reliability of this interview schedule was 5 assessed when 153 respondents (90 women and $63^{-}$ men) in a population survey were re-interviewed after 12 months by a different team of interviewers. Table II shows that two respondents previously $\overrightarrow{0}$ classified as fit and nine previously classified ogs:impaired only were reclassified as disabled. The diagnoses in these nine impaired cases were chroffic bronchitis (3), cerebrovascular disease (2), rheuma $\vec{a}-\frac{\partial}{0}$ toid arthritis, rheumatic heart disease, muscularo dystrophy, and ulcerative colitis. In all these cases there was indirect evidence in terms of hospital attendances and general practitioner consultations $\frac{}{3}$ to indicate that their condition had worsened during the intervening year. The diagnoses in the two res-0 pondents previously classified as fit and becoming disabled during the year were epilepsy and depression.

Twelve respondents originally classified as disabled 3 . were reclassified as impaired. In nine of these cases who suffered from prolapsed intervertebral disc 3 (3), arthritis (2), Parkinson's disease, epilepsy, chronic bronchitis, and internal derangement of a knee joint, there was evidence to support the change. For instance, the woman suffering from Parkinson's disease had undergone surgery with remark- $-\infty$ ably good effect. In three cases, however, whoN suffered from rheumatic heart disease, angina, and N bronchiectasis, there was nothing recorded in theointerview schedule to explain the change in classification. Seven respondents showed a change in assessment of severity of disability, three improving? and four worsening. In all seven these findings were in respondents suffering from conditions in which theo disability would be expected to fluctuate. 
Complete agreement between assessments occurred in $122(80 \%)$ people. Agreement in the identification of the presence or absence of disability occurred in $130(85 \%)$ people. In only three out of 153 reassessments after an interval of one year did change occur which could not be explained and these levels of repeatability were considered satisfactory.

\section{Discussion}

In a previous report we described the use of the interview schedule for the second stage of a largescale population survey with a sample of 1,142 adults aged 35 to 74 (Bennett, Garrad, and Halil 1970). The results showed central estimates of $7.2 \%$ men and $9.7 \%$ women disabled according to the definition and criteria stated.

As preparation for the field work of this study a training programme was organized lasting approximately three weeks for each new interviewer and they were trained in small groups. The training consisted of three stages. First, the principles on which the schedule had been developed were carefully explained and the method of administration was demonstrated in simulation role-playing interviews by the two of us. Then the interviewers took it in turns to interview each other in our presence and their performance was commented on. Secondly, they practised the interview on inpatients and outpatients, again under observation by one of us. Finally, they completed a series of interviews on selected outpatients in their homes. Each interview schedule was scrutinized by one of us for completeness and internal consistency and the data were discussed in detail with the interviewer. When necessary, these were checked with hospital records or with the patient. During the survey, a similar procedure to the last stage of training was followed. Each schedule was scrutinized on the day following the interview and, when necessary, omissions were completed by a repeat visit. Schedules were discussed with the interviewers to cross-check on accuracy and to minimize variation between interviewers and interviewer. drift. Seventeen interviewers took part in the field work which lasted for just over three months.

The second stage of the study was restricted to persons aged 35 to 74 years. Below this age the number of disabled in the first stage sample was too small for estimates of prevalence to be made. By contrast, over the age of 74 the proportion of those functionally disabled rose very sharply, reflecting how the processes of increasing severity of disease with age restrict function and result in dependence.

This illustrates the dynamic nature of this con- ceptual model of disability. An infant born 'fit' and continuing to survive will progress to become impaired and subsequently disabled. The usual sequence of events is for the period of 'fitness' to end in middle age or later with the manifestation of one or more of the chronic degenerative diseases, most commonly of locomotor or internal origin. As the disease progresses the individual loses his independence of living and thereafter becomes increasingly disabled. However, for each individual the time periods of the different stages will vary. Following the onset of diseases such as rheumatoid arthritis or multiple sclerosis, the period of disability may extend to many years, whereas after a severe stroke or the onset of some malignant condition the period may be short. An infant born with a congenital abnormality is impaired and possibly disabled throughout life. A child involved in an accident may lose a limb and be impaired for life but only becomes disabled when the effects of ageing supervene. Death is an event which at some point intervenes to prevent further progression. Acute illness with cure or recovery from a chronic condition both show how the model need not be considered unremittingly progressive, even in the short term.

The critical point in the progression is when independence of living is lost and disability ensues. Obviously this is not as sharp a point of demarcation in all cases as would be desired for the purposes of measurement. If acute illness is excluded by setting a minimum time qualification, such as three months, factors causing oscillation at this point are changes in severity of the disease or changes in the psychological state of the patient. If these are liable to fluctuate then the individual's ability to perform tasks unaided may vary. Table II shows that by far the largest number of individuals who were reclassified one year later were changed from the minimally disabled to the group of impaired only or vice versa.

However, in the same way as fluctuation or remission in the severity of a disease process allows independence to be regained, so may treatment or the provision of aids or gadgets restore the necessary function. Thus a simple dressing aid or walking stick may restore enough function to allow independence. These may, however, be required in addition to a major operation such as hip arthroplasty. The objectives of the surgical procedure or use of the gadgets are all similar in attempting to restore and maintain the ability of the individual to function independently, or to function to the best of limited abilities. It is not difficult to refer these same objectives to the practice of internal medicine or psychiatry or even to offer them as the objectives for 
the practice of medicine. For in many of the chronic conditions presently treated, progression of the disease is largely unaffected. Functional evaluation of the effectiveness of many widely used treatments or procedures is required.

The present schedule was not designed for use in the clinical situation. Furthermore, the problem of secondary gain, which is minimal in the research setting, may prove of some importance when using such a schedule for clinical assessments. However, no method will overcome this or all other possible disadvantages. Further work is now examining whether the coarse scaling of this instrument designed for epidemiological surveys can be refined to extend its usefulness into the area of evaluating the outcome and effectiveness of various treatments.

\section{SUMmaRY}

A concept of disability is put forward based on appreciation of inability to perform essential activities of daily living. Inability to perform these activities unaided necessitates dependence on another person and the severity of disability is proportional to the degree of dependence. A review of published evidence suggests that the interview is the method of choice for population surveys to study the epidemiology of disability as defined. The development of sections of an interview schedule designed to assess disability and identify the associated impairment category and diagnostic group is described. Comparison of data obtained by trained interviewers using the schedule with data recorded in the clinical record showed levels of agreement between 90 and $100 \%$. For a series of assessments repeated after an interval of one year, agreement was found in $80 \%$ but in only $2 \%$ were the changes inexplicable.

These studies were supported by the Endowment Funds of St. Thomas' Hospital, the Department of Health and Social Security, the South-West Metropolitan Regional Hospital Board, and the City Parochial Charities. We wish to thank Professor W. W. Holland for advice and encouragement; Drs. T. C. Picton Williams and D. A. H. Yates for generous help and permission to study their patients; Miss S. Smeeton for secretarial support; and all members of the department who assisted us.

\section{REFERENCES}

Bennetr, A. E., Garrad, J., and Halil, T. (1970). Chronic disease and disability in the community: a prevalence study. Brit. med. J., 3, 762.

CanNell, C. F., and Marquis, K. H. (1967). Effect of some experimental interviewing techniques on reporting in the Health Interview Survey. Survey Research Center, University of Michigan. study of the effects of reinforcement, question length and re-interviews on reporting selected chronicz conditions in household interviews. Survey Research $b$ Center, University of Michigan.

Cole, S. L., and Griffith, G. C. (1958). Assay o $\overrightarrow{\vec{E}}$ antianginal agents-the rapport period. J. Amer. med 5 Ass., 168, 275.

Colombotos, J., Elinson, J., and Loewenstern, R (1968). Effect of interviewers' sex on interview res? ponses. Publ. Hlth Rep. (Wash.), 83, No. 8, 685.

Committee on the Medical Rating of Physicaten IMPAIRMENT (1958). A guide to the evaluation of permanent impairment of the extremities and back: J. Amer. med. Ass., 166, Feb. 15th, Special edition.

EKWALL, B. (1966). Method for evaluating indications for rehabilitation in chronic hemiplegia. Acta med. scand. Suppl. 450.

Foulds, G. A. (1965). Personality and Personal Illnessî Tavistock Publications, London.

JefFerys, M., Millard, J. B., Hyman, M., and Warrenso M. D. (1969). A set of tests for measuring motor impairment in prevalence studies. J. chron. Dis., 22\% 303.

KaTZ, S., Ford, A. B., Moskowitz, R. W., JACKSONz B. A., and JAFFE, M. W. (1963). Studies of illness is the aged. J. Amer. med. Ass., 185, 914.

Kelman, H. R., and Willner, A. (1962). Problemsoin measurement and evaluation of rehabilitation. $A$ re $h$. phys. Med., 43, 172.

LiTMAN, T. J. (1962). The influence of self conception 奥高 life orientation factors in the rehabilitation of th orthopedically disabled. J. Hlth hum. Behav., 3, 249.

(1964). An analysis of the sociologic factor affecting the rehabilitation of physically handicapped patients. Arch. phys. Med., 45, 9.

MEDICAL RESEARCH CounCIL (1966). A questionnaire on respiratory symptoms approved by the M.R.C. Committee on Research into Chronic Bronchitis.

MULLER, J. N. (1961). Rehabilitation evaluation-somes social and clinical problems. Amer.J.pub. Hlth, 51, 403 ?

OsGOOD, C. E., and SuCI, G. J. (1952). A measure of relation determined by both mean difference an $\Phi$. profile information. Psychol. Bull., 49, 251.

ROSE, G. A. (1962). The diagnosis of ischaemic hearo pain and intermittent claudication in field surveys Bull. Wld Hlth Org., 27, 645.

SETT, R. F. (1963). Simplified tests for evaluation of patients with chronic illness (cerebro-vascular acc은. dents). J. Amer. Geriat. Soc., 11, 1095.

SHONTZ, F. C., and FINK, S. L. (1957). The significance of patient-staff rapport in the rehabilitation of indis viduals with chronic physical illness. J. consuln్ Psychol., 21, 327.

TAMERIN, J. S. (1964). The perception of progress in rehabilitation. Arch. phys. Med., 45, 17.

WoLF, S. (1950). Effects of suggestion and conditioning on the action of chemical agents in human subjects: the pharmacology of placebos. J. clin. Invest., 29, 1000

WYLIE, C. M., and WHITE, B. K. (1964). A measure of. disability. Arch. environm. Hlth, 8, 834. 